\title{
CARACTERIZAÇÃO DE BOLHAS COM USO DE ESPUMANTE MIBC E ALFA-TERPINEOL EM COLUNA DE FLOTAÇÃO APLICANDO TÉCNICA DE HYDROMESS
}

\author{
Moacir Medeiros Veras \\ PPGEMinas - Departamento de Eng. de Minas - UFPE e Lab. Processamento Mineral - \\ Instituto Federal do RN, Bolsista DTI-III do CNPq - Projeto FINEP - Fortalecimento da \\ Estrutura de Apoio à Pesquisa para o APL Mineral RN, \\ moacir@cefetrn.br

\section{Carlos Adolpho Magalhães Baltar} \\ Engenheiro de Minas, M.Sc., D.Sc., Departamento de Engenharia de Minas. Centro de \\ Tecnologia e Geociências. Universidade Federal de Pernambuco. Rua Acadêmico Hélio \\ Ramos, s/n. Cidade Universitária. - Recife/PE. CEP 50440-530, camb@ufpe.br

\section{João Bosco de Araújo Paulo} \\ Prof. Depto de Engenharia Química - Universidade Federal do Rio Grande do Norte \\ jbosco@eq.ufrn.br \\ José Yvan Pereira Leite \\ Prof. do Instituto Federal do Rio Grande do Norte - Lab. de Processamento Mineral \\ leite@cefetrn.br
}

\section{RESUMO}

No processo de flotação, empregado na separação seletiva com base nas propriedades superficiais das espécies minerais, o contato entre as bolhas de ar e as partículas dispersas tem papel fundamental. O Tamanho das bolhas pode variar devido ao fenômeno da coalescência, sobre o qual tem influência a concentração do reagente espumante, resultando em uma não uniformidade do diâmetro das bolhas. Para entender melhor este comportamento, estudou-se a influência da concentração do metil-isobutil-carbinol (MIBC) e alfa-terpineol (ÓLEO DE PINHO) no diâmetro médio das bolhas. Para a medição do tamanho das bolhas, utilizou-se uma nova metodologia adaptada de uma técnica, desenvolvida pela TUClausthal (Technische Universität Clausthal) Alemanha, que faz uso da combinação de um capilar de vidro e uma sonda fotoelétrica que pode caracterizar as bolhas em termos de distribuição volumétrica, superficial e numérica, com base no índice de refração das fases presentes. Os experimentos foram realizados com água destilada e espumante cuja concentração variou de 0 a 16,3 ppm. Os diâmetros das bolhas apresentaram tamanhos variando de 2,27 a 2,67 mm em sistema ar/água. Para a determinação dos diâmetros das bolhas é necessário que o sistema forme bolhas compatíveis com os limites de dimensões dos capilares de vidro dispostos pelo equipamento. O método empregado para medida do diâmetro de bolhas de ar pelo índice de refração representa uma alternativa aos métodos de análise de imagem aplicados em estudos similares.

PALAVRAS-CHAVE: caracterização de bolha, flotação, espumantes, mibc, alfaterpineol. 


\section{CHARACTERIZATION OF BUBBLES WITH USE OF SPARKLING MIBC AND ALPHA-TERPINEOL COLUMN FLOTATION OF APPLYING TECHNIQUE HYDROMESS}

In the flotation processes, used in the selective separation based on the surface properties of minerals, the contact between the bubble of air and dispersed particles has basic role. The bubbles size can vary due the phenomenon of coalescence, resulting in an heterogeneity of the bubbles diameters. To understand better this behavior, this work presents a new methodology for bubble's air characterization to the flotation process when using a column. For measuring the size of the bubbles, we used a new methodology adapted from a technique developed by TUClausthal (Technische Universität Clausthal) Germany, we use a combination of a glass capillary and photoelectric probe which based on the difference of index of refraction of both phases leads to characterize the bubble in terms of distribution of volume, area and/or number. The diameters of the analyzed bubbles presented a distribution of size varying from 2.27 to $2.67 \mathrm{~mm}$ to the system air / water. The experiments were carried out using distilled water and froth agent in the concentration of 0 to $16.3 \mathrm{ppm}$. These diameters can be measured only when the system forms compatible bubbles with the limiting dimensions of the glass capillary arranged in the equipment. The method employed for measure the bubbles air diameters based on the index of refraction represents an alternative to the conventional image analysis method applied in similar systems.

KEY-WORDS: characterization of bubble, flotation, foaming, mibc, alfa-terpinel. 


\section{CARACTERIZAÇÃO DE BOLHAS COM USO DE ESPUMANTE MIBC E ALFA-TERPINEOL EM COLUNA DE FLOTAÇÃO APLICANDO TÉCNICA DE HYDROMESS}

\section{INTRODUÇÃO}

A técnica de flotação é empregada na separação seletiva de minerais com base nas diferenças existentes entre as propriedades superficiais das espécies minerais. A eficiência da separação depende fortemente das condições em que ocorrem os contatos entre as bolhas e as partículas, etapa inicial e imprescindível para a flotação (Baltar, 2008). A freqüência das colisões aumenta com a redução do tamanho das bolhas, devido ao aumento da área superficial disponível para o contato com as partículas.

Neste sentido, o fenômeno da coalescência das bolhas é prejudicial já que implica em aumento do diâmetro médio das bolhas. Uma das funções do reagente espumante é a de dificultar a coalescência. Uma pequena adição do espumante tem forte influência no tamanho das bolhas (Grau et al, 2005), cujo diâmetro médio tende a diminuir até o tamanho original (Grau e outros, 2005; Azgomi e outros, 2007), A concentração correspondente a esse tamanho limite é denominada concentração crítica de coalescência (ccc) que é característica de cada sistema (Cho e Laskowski, 2002).

O uso de espumantes em processos de flotação está relacionado com a necessidade de se reduzir a tensão superficial, evitar a coalescência e facilitar a adesão das partículas hidrofóbicas às bolhas de ar (Baltar, 2008). O MIBC e POLIGLICOL são espumantes amplamente usados em processos de flotação de minerais diversos (Sampaio e outros, 2001).

Os espumante, de modo geral, são compostos heteropolares que contêm grupo de radicais $\mathrm{OH}, \mathrm{COOH}, \mathrm{CO}, \mathrm{OSO}_{2}$ e $\mathrm{SO}_{2} \mathrm{OH}$, ligados a uma cadeia hidrocarbônica capaz de concentrar-se na interface ar/água. Caracterizados por uma extremidade hidrofílica ou polar direcionada para a fase da água, e uma extremidade hidrofóbica ou apolar direcionada para a fase gasosa (Bulatovc, 2007). O reagente forma uma monocamada de moléculas na interface líquido-gás reduzindo a tensão interfacial estabilizando a bolha e evitando a coalescência (Gourram-Badri e outros, 1997).

$\mathrm{O}$ metil-isobutil-carbinol (MIBC) - $\mathrm{C}_{6} \mathrm{H}_{14} \mathrm{O}$ e o alfa-terpineol (óleo de pinho) - $\mathrm{C}_{10} \mathrm{H}_{18} \mathrm{O}$ são muito empregados na flotação de minérios metálicos contendo minérios argilosos. A desvantagem dos espumantes alifáticos é que a sua composição não é sempre constante e, conseqüentemente, as propriedades da espuma são variáveis (Bulatovc, 2007).

Diversos autores têm mostrado a importância do controle do tamanho das bolhas em um processo de flotação (Haris, 1982; Xia e Peng, 2007). Apesar da importância do tema a dificuldade para se monitorar a variação do tamanho das bolhas, em uma célula de flotação, tem limitado o avanço do conhecimento relacionado à coalescência na flotação.

No trabalho utilizou-se uma técnica desenvolvida pela TUClausthal (Technische Universität Clausthal) da Alemanha que faz uso de uma combinação capilar/ sonda fotoelétrica para capturar as bolhas e medir o sinal através dos índices de refração das fases. Através do sinal e análise estatística é possível determinar o diâmetro das bolhas em densidade numérica, área e volumétrica segundo manual do Hydromess 1.40 (1996). 
A sonda consiste em um conjunto composto por um capilar de vidro, duas células luminosas e dois fototransistores. As bolhas são capturadas pelo capilar de vidro e transformadas em elipsóides cilíndricos como pode ser observado na Figura 1.

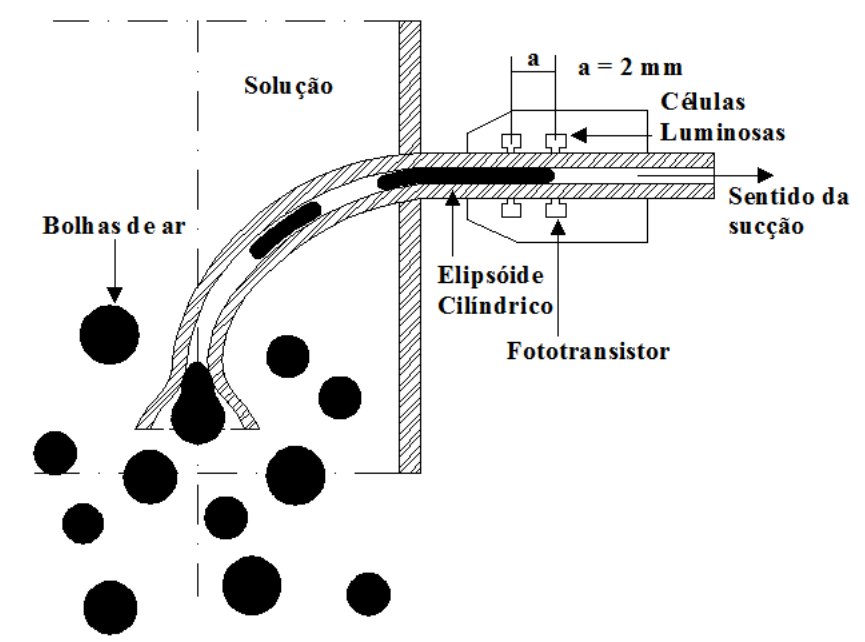

Figura 1. Sonda de sucção fotoelétrica para captura de bolhas de ar e formação de elipsóide no capilar de vidro que será registrado pelo fototransistor

A Figura 2 exemplifica uma partícula de ar deformada no capilar sem capa de filme da fase contínua e um sinal de medida idealizado no fototransistor. O limiar idealizado são equivalentes à metade da esfera, de um lado um certo volume da fase contínua é medido com a elipse porque a sonda registra somente o comprimento de um cilindro que é marcado pelo gatilho de ajuste que regula o sinal para fazer os volumes das duas extremidades equivalentes.

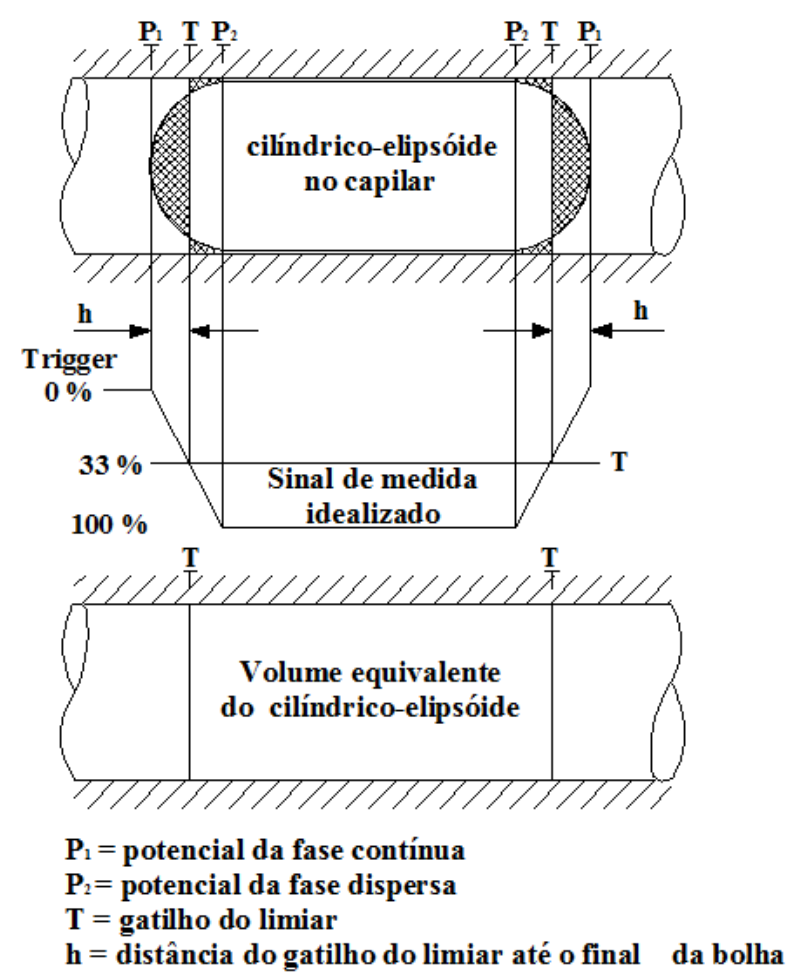

Figura 2. Partícula de ar deformada no capilar para leitura com sinal idealizado do cilindro-elipsóide. 
Quando o elipsóide cilíndrico passa pelos feixes das células luminosas provoca uma perturbação no sinal que está fazendo a leitura do índice de refração da fase contínua, dois fototransistores detectam o início e final da interferência do sistema. O sinal bruto é tratado e convertido em sinal quadrado para determinar o tempo de passagem do elipsóide cilíndrico diante das células $1\left(\mathrm{t}_{1}\right)$ e $2\left(\mathrm{t}_{2}\right)$. A possível decalagem entre os sinais gerados pelas células 1 e 2 é designada pelos tempos $t_{3}$ e $t_{4}$ respectivamente, conforme apresentado na Figura 3.

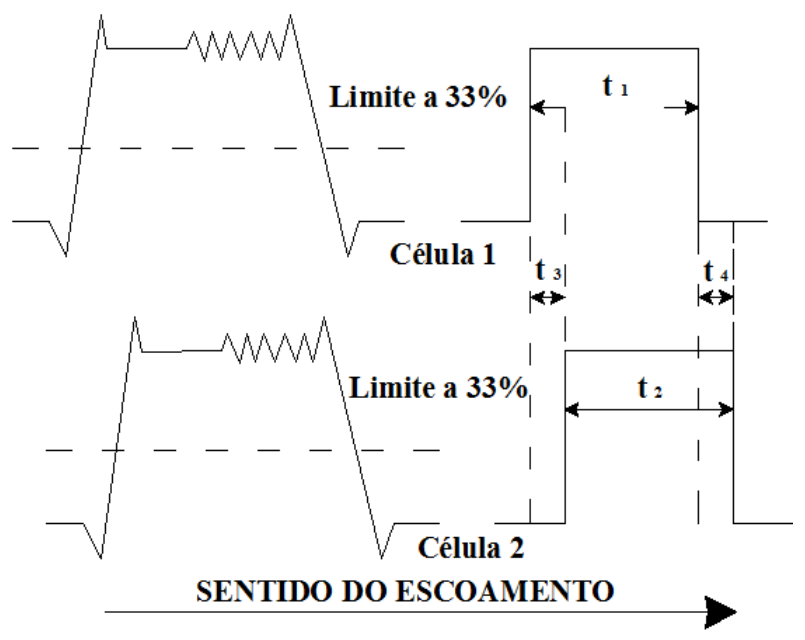

Figura 3. Leitura do sinal bruto e transformação em sinal quadrado em (b) (Fonte:

Paulo, 2003).

\section{EXPERIMENTAL}

Para caracterizar o diâmetro de bolhas, faz-se uso do aparato experimental mostrado na Figura 2 e do software de aquisição Hydromess ${ }^{\circledR} 1.40$. Utiliza-se uma bomba de vácuo que tem como finalidade de manter a velocidade de sucção no interior do capilar estabilizada.

Segundo Paulo (1996), a velocidade de sucção da sonda ( $U_{a s p 1}$ e $U_{a s p 2}$ ) pode ser calculada pela equação (1). No caso ideal em que $t_{3}$ e $t_{4}$ (Figura 1-b), as velocidades de sucção são iguais. Desta forma quando o elipsóide cilíndrico formado no capilar de vidro apresentar intervalo conveniente a sonda pode determinar o comprimento $(l)$ pela equação (2).

$U_{\text {asp1 }}=\frac{a}{t_{3}}$ e $U_{\text {asp } 2}=\frac{a}{t_{4}}$

equação (1)

$l=U_{\text {aspl }}, t_{1}$ ou $l=U_{\text {asp }}, t_{2}\left(\right.$ parat $\left._{1}=t_{2}\right)$

Onde:

$a$ = distância entre as duas células luminosas;

$t_{3}$ e $t_{4}=$ obtidos a partir das diferenças dos tempos $t_{1}$ e $t_{2}$ entre as duas células (conforme figura 1-b)

l = comprimento do elipsóide cilíndrico (gota deformada no interior do capilar);

$t_{1}$ e $t_{2}=$ tempo de passagem do elipsóide cilíndrico diante das células luminosas 1 e 2

Se o elipsóide cilíndrico preencher a secção transversal do capilar, o diâmetro da esfera equivalente ao volume da partícula $\left(d_{v}\right)$ que possui o mesmo volume do elipsóide cilíndrico pode ser calculado pela equação (3), onde $\mathrm{d}_{\text {cap }}$ corresponde ao diâmetro interno do capilar. 
$d_{v}=\sqrt[3]{\frac{3}{2}} \mathrm{~d}_{\text {cap }}^{2} l$

equação (3)

O aparato experimental faz uso de um computador com software do equipamento, Hydromess ${ }^{\circledR}$ 1.40, sonda fotoelétrica, capilar de vidro, coluna de acrílico com cerâmica porosa, quitazato e bomba de vácuo. Os detalhes do aparato podem ser apreciados na Figura 2.

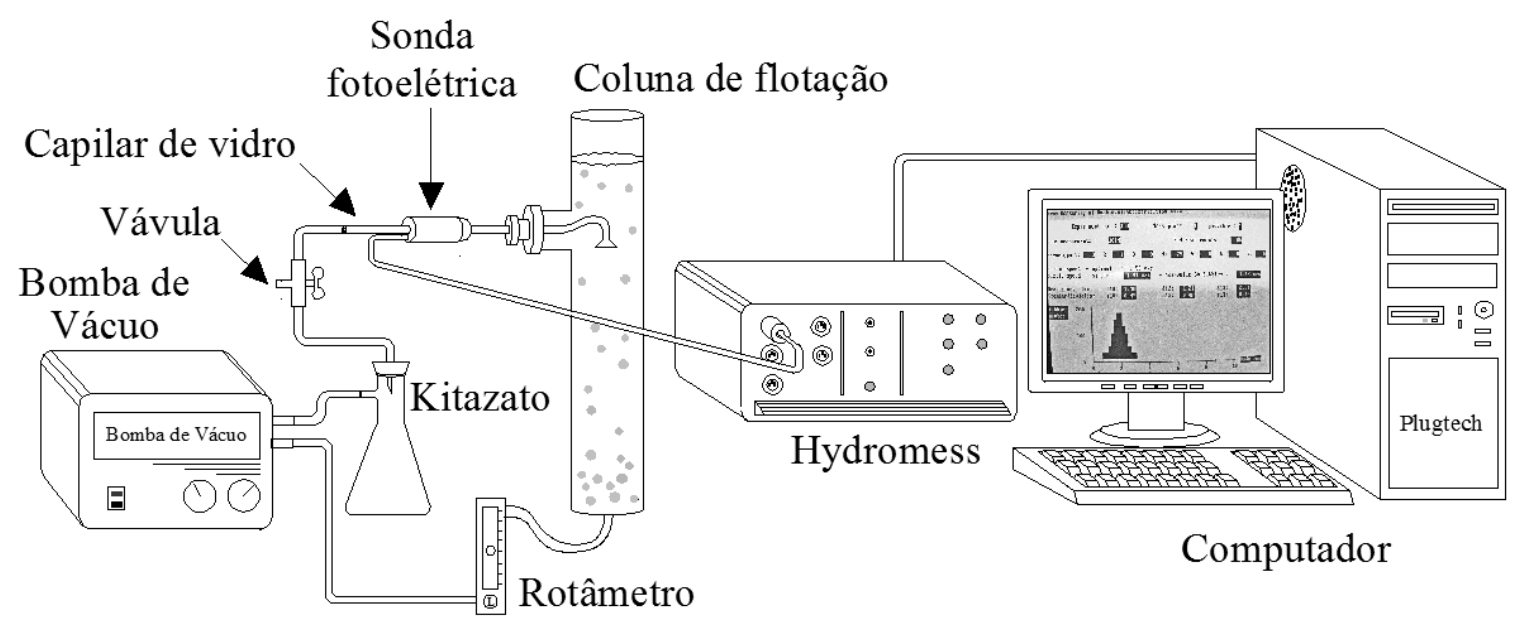

Figura 2. Aparato para medida de diâmetro de bolha em coluna de flotação.

As análises de diâmetro de bolhas foram feitas com todas as soluções em água destilada visando simular condições padrões. Foram utilizados dois reagentes para medida de tamanho de bolha visando comparar os comportamentos.

- Os capilares de vidro utilizados na sonda possuem diâmetro interno de 0,6 e 0,8 mm;

- A sonda fotoelétrica foi calibrada em modo automático pelo software;

- A velocidade de sucção foi ajustada em valores próximos de 1,5 m/s com auxílio de bomba de vácuo;

- A vazão de ar se manteve constante em $101,8 \mathrm{~cm}^{3} / \mathrm{min}$. para todos os ensaios;

- Os experimentos foram executados usando o reagente MIBC e alfa-terpineol;

- As concentrações do espumante variaram de 0 a 16,3 ppm.

\section{RESULTADOS E DISCUSSÕES}

O programa Hydromess ${ }^{\circledR} 1.40$ gera resultados da distribuição de diâmetro de bolha em termos de densidade numérica, superficial e volumétrica. Os resultados são apresentados em diâmetro de Sauter, pois correlaciona superfície/volume da partícula.

O estudo apresenta resultados da variação do diâmetro de bolhas usando dois espumantes que são metil-isobutil-carbinol e alfa-terpineol. Os dados do diâmetro de Sauter podem ser observados na Tabela 1. 
Tabela 1. Diâmetro médio obtido a partir de várias funções de distribuições (vazão de ar constante e igual a $101,8 \mathrm{~cm}^{3} / \mathrm{min}$ ).

\begin{tabular}{|c|c|c|c|c|}
\hline \multirow[t]{2}{*}{ - $\quad$ ppm } & \multicolumn{3}{|c|}{$\begin{array}{l}\text { - MIBC } \\
\text { - ALFA-TERPINEOL }\end{array}$} & \multirow[t]{2}{*}{ - $\mathrm{S}$} \\
\hline & $d_{1,0}$ & $d_{1,2}$ & $d_{1,3}$ & \\
\hline \multirow{2}{*}{0,0} & 2,23 & 2,44 & 2,55 & 0,21 \\
\hline & 2,18 & 2,39 & 2,51 & 0,20 \\
\hline \multirow{2}{*}{1,6} & 2,09 & 2,52 & 2,67 & 0,25 \\
\hline & 1,31 & 3,01 & 3,34 & 0,30 \\
\hline \multirow{2}{*}{3,3} & 2,03 & 2,29 & 2,45 & 0,25 \\
\hline & 1,34 & 2,56 & 2,70 & 0,28 \\
\hline \multirow{2}{*}{4,9} & 2,02 & 2,28 & 2,43 & 0,25 \\
\hline & 1,24 & 2,63 & 2,82 & 0,19 \\
\hline \multirow{2}{*}{6,5} & 1,95 & 2,29 & 2,43 & 0,26 \\
\hline & 1,15 & 2,30 & 2,39 & 0,20 \\
\hline \multirow{2}{*}{8,2} & 1,12 & 2,33 & 2,49 & 0,25 \\
\hline & 2,08 & 2,23 & 2,32 & 0,20 \\
\hline \multirow{2}{*}{9,8} & 1,26 & 2,17 & 2,29 & 0,25 \\
\hline & 2,06 & 2,21 & 2,31 & 0,18 \\
\hline \multirow{2}{*}{11,4} & 0,86 & 2,15 & 2,27 & 0,26 \\
\hline & 2,04 & 2,18 & 2,25 & 0,19 \\
\hline \multirow{2}{*}{13,1} & 1,04 & 2,12 & 2,33 & 0,26 \\
\hline & 2,05 & 2,24 & 2,27 & 0,18 \\
\hline \multirow{2}{*}{14,7} & 0,99 & 2,18 & 2,37 & 0,26 \\
\hline & 2,01 & 2,14 & 2,21 & 0,30 \\
\hline \multirow{2}{*}{16,3} & 0,99 & 1,77 & 2,47 & 0,34 \\
\hline & 1,99 & 2,13 & 2,20 & 0,30 \\
\hline
\end{tabular}

\section{Legenda}

S - desvio padrão;

$\mathrm{d}_{1,0}$ - diâmetro médio obtido a partir da distribuição da densidade em número de bolhas;

$\mathrm{d}_{1,2}$ - diâmetro médio obtido a partir da distribuição da densidade em área de bolhas;

$\mathrm{d}_{1,3}$ - diâmetro médio obtido a partir da distribuição da densidade em volume de bolhas.

O gráfico apresentado na Figura 3 mostra a variação do diâmetro médio de bolhas com concentrações do MIBC e ALFA-TERPINEOL variando entre 0 à 16,3 ppm. Os valores são expressos para as distribuições de densidade em volume $\left(\mathrm{q}_{3}\right)$. Pode-se notar que o diâmetro das bolhas apresenta maior variação em baixas concentrações onde a diferença no diâmetro de bolhas é de 0,67 mm na concentração 1,6 ppm. Para essa mesma concentração pode-se notar que o MIBC tende a um aumento de 5 \% em relação ao diâmetro de bolha sem aditivo, e no ALFA-TERPINEOL as bolhas tendem a sofrer um aumento da ordem de 30 \%. Em concentrações acima de 1,6 ppm os dois reagentes tendem a apresentarem diâmetros de bolhas de mesmo tamanho. 


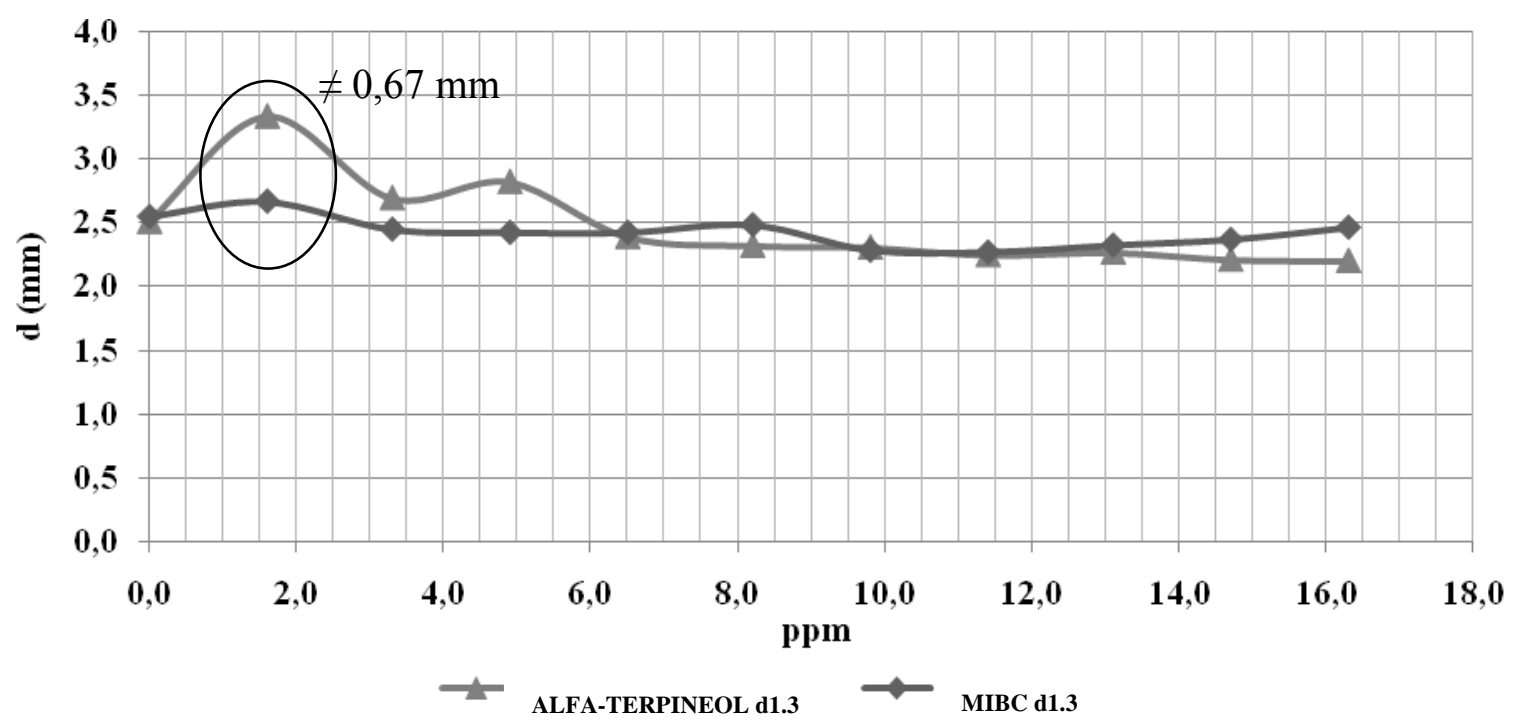

Figura 3. Distribuição do diâmetro de bolhas em função da variação de concentração do espumante.

A equação (4) apresenta a função $q_{3}$ da distribuição de densidade em volume de bolhas.

$q_{3}\left(d_{j}\right)=\frac{d_{j}^{3} N_{j}}{\Delta d \sum_{j=1}^{M} d_{j}^{3} N_{j}}$ equação (4)

Onde:

$d_{j}=$ diâmetro médio de um intervalo de classes de gotas j;

$N_{j}=$ número de gotas da classe j;

$\Delta d=$ variação dos diâmetros extremos da classe j;

$M=$ número de classes.

A Figuras 4, apresenta exemplo dos resultados de uma análise realizada com Hydromess 1.40 em termos de q q $_{3}$ para as concentrações do MIBC e ALFA-TERPINEOL em 10 ppm respectivamente com desvio padrão (s), $\mathrm{d}_{50}$ e o erro (error). Os gráficos mostram curvas que representam os diâmetros médios obtidos a partir da distribuição da densidade em número de bolhas $\left(\mathrm{d}_{1,0}\right)$, diâmetro médio obtido a partir da distribuição da densidade em área de bolhas $\left(\mathrm{d}_{1,2}\right)$ e o diâmetro médio obtido a partir da distribuição da densidade em volume de bolhas $\left(\mathrm{d}_{1,3}\right)$. Os gráficos proporcionam um melhor entendimento do comportamento da distribuição do tamanho das bolhas para os dois reagentes quando em operação no Hydromess.

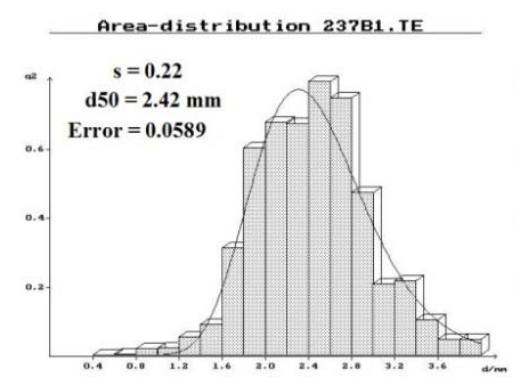

(a)

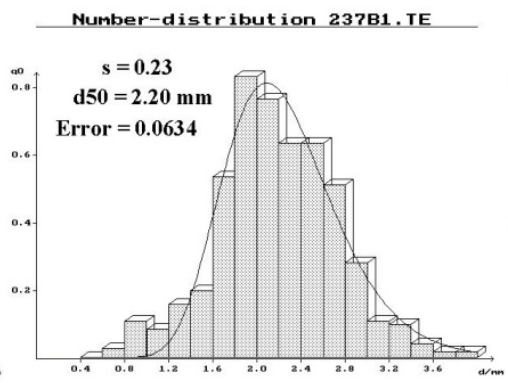

(b)

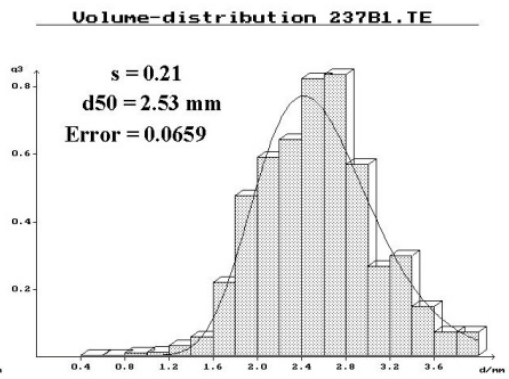

(c) 


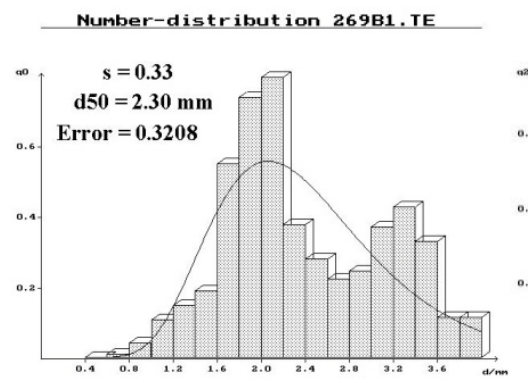

(d)

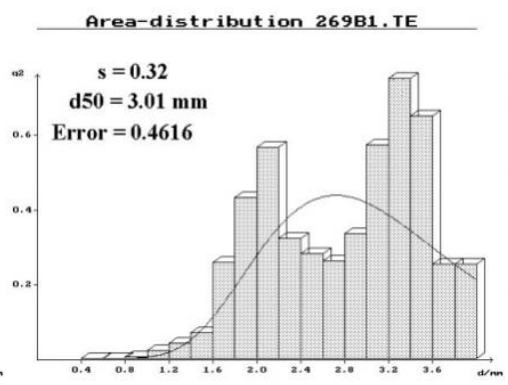

(e)

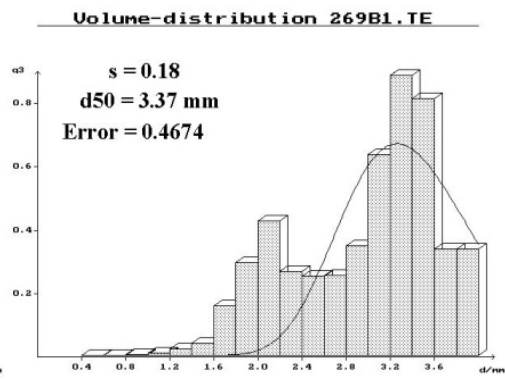

(f)

Figura 4. Curva de distribuições de densidade em área, número e volume com as concentrações em 10 ppm para o reagente MIBC (a), (b), (c) e ALFA- TERPINEOL (d), (e), (f).

Embora a determinação de diâmetro de bolhas por meio da técnica combinada capilar/sonda foto elétrica e o software Hydromess ${ }^{\circledR} 1.40$ apresente boa precisão, porém a operacionalidades do equipamento é criteriosa. Pode-se citar o diâmetro estimado da bolha que deve ser no mínimo três vezes maior que o diâmetro interno do capilar e não ultrapassar $15 \mathrm{~mm}$ de comprimento do elipsóide cilíndrico, a velocidade de sucção ideal deve ser da ordem de $1,5 \mathrm{~m} / \mathrm{s}$. O sistema não apresenta resultados significantes quando aplicado em células mecânicas.

\section{CONCLUSÃO}

Observou-se que a técnica combinada capilar/sonda fotoelétrica e o software Hydromess ${ }^{\circledR}$ 1.40 permite a medição o tamanho de bolhas geradas em uma coluna de flotação. Com uma vazão de ar em 101,8 $\mathrm{cm}^{3} / \mathrm{min}$. e na ausência de espumante, o diâmetro médio das bolhas é de 2,51 mm,.

Os resultados mostraram uma relação entre o diâmetro médio das bolhas e a concentração do MIBC e o ALFA-TERPINEOL. A concentração dos espumante em 1,6 ppm provoca uma diferença no diâmetro de bolha de 20 \% entre os reagentes, 5 \% entre o MIBC e a solução sem aditivo e 30 \% entre o ALFA-TERPINEOL e a solução sem aditivo, respectivamente. As diferenças tendem a desaparecer para concentrações acima da de 1,6 ppm.

O efeito do tipo de espumante, no caso da comparação do MIBC com o alfa-terpinol é perceptível apenas para baixas concentrações. Para concentrações maiores o diâmetro apresentados pelas bolhas tendem a coincidir independentemente do espumante testado.

\section{AGRADECIMENTOS}

Os autores gostariam de expressar seus agradecimentos ao IFRN, CNPq - FINEP FAPERN (Projeto Fortalecimento da Estrutura de Apoio à Pesquisa para o APL Mineral do Rio Grande do Norte) pela concessão da bolsa.

\section{REFERÊNCIAS BIBLIOGRÁFICAS}

1. Azgomi, F., Gomez, C.O., Finch, J.A., Correspondence of gas holdup and bubble size in presence of different frothers, International Journal of Mineral Processing, v. 83, p. 1-11, 2007. 
2. Baltar, C.A.M., Flotação no Tratamento de Minério, Recife-PE, UFPE, Brasil, p. 114-131, 2008.

3. Bulatovc, S.M., Handbook of flotation reagents, Chemistry, Theory and Practice: Flotation of Sulfide Ores, Elsevier Science \& Technology Books, v. 1, 2007.

4. Cho, Y.S., Laskowski, J.S., Effect of flotation frothers on bubble size and foam stability, International Journal of Mineral Processing, v. 64, p. 69-80, 2002.

5. Gourram-Badri, F., Conil, P., Morizot G., Measurements of selectivity due to coalescence between two mineralized bubbles and characterization of MIBC action on froth flotation, International Journal of Mineral Processing, v. 51, p. 197-208, 1997.

6. Grau, R.A.; Laskowski, J.S.; Heiskanen, K., 2005. Effect of frothers on bubble size. International Journal of Mineral Processing, 76, 225-233, 2005.

7. Harris, P.J., Frothing Phenomena and Frothers. In.: Principles of Flotation. King, R.P. (Editor). South African Institute of Mining and Metallurgy, Johannesburg. Capítulo 13, 237-250, 1982.

8. Manual Hydromess 1.40, Equipamento de Medição para a Derterminação de Parâmetros Fluido Dinâmicos em Fluxo Multi-Fásicos, Technocon GmbH, Tannenhöhe, Claustal-Zellerfeld, Germany, 2002.

9. Paulo, J. B. A. - Operação otimizada de um novo misturador-decantador à inversão de fases: aplicação à extração de cobre (título traduzido do francês). Toulouse-França, julho, Tese de doutorado. Instituto Nacional Politécnico de Toulouse. p.125, 1996.

10. Paulo, J.B.A.,Fernandes Jr., W.E., Moraes, N.A., Souza, E.M., Lira, B.B., Medida de Tamanho e Distribuição de Gota em Novo Desing de Extrator Líquido-Líquido, Metrologia para a vida, Sociedade Brasileira de Metrologia (SBM), Recife-PE, Brasil, 2003.

11. Xia, Y.K,; Peng, F.F., Selection of Frothers from Residual Organic Reagents for Copper-Molybdenite Sulfide Flotation. International Journal of Mineral Processing, 83, 68-75, 2007. 